\title{
Belief-Based Packet Forwarding in Self-Organized Mobile Ad Hoc Networks with Noise and Imperfect Observation
}

\author{
Zhu (James) Ji, Wei Yu, and K. J. Ray Liu \\ Electrical and Computer Engineering Department and Institute for Systems Research \\ University of Maryland, College Park, MD 20742 \\ email: zhuji, weiyu, kjrliu@umd.edu
}

\begin{abstract}
In self-organized mobile ad hoc networks (MANET) where each user is its own authority, fully cooperative behaviors, such as unconditionally forwarding packets for each other, cannot be directly assumed. In this paper, we focus on cooperation enforcement in the self-organized mobile ad hoc networks with noise and imperfect observation and study the basic packet-forwarding function using the repeated game models with imperfect information. A belief-based packet forwarding framework is proposed to obtain cooperation-enforcement strategies solely based on each node's own past actions and its private imperfect observation of other nodes' information. The simulation results illustrate that the proposed belief-based packet forwarding approach can enforce the cooperation with only a small performance degradation compared to the unconditionally cooperative outcomes in the ad hoc networks with noise and imperfect observation.
\end{abstract}

\section{INTRODUCTION}

Mobile ad hoc networks (MANET) have drawn extensive attention in recent years due to the increasing demands of its potential applications [1], [2]. In traditional emergency or military situations, the nodes in a MANET usually belong to the same authority and act cooperatively for the common goals. Recently, emerging applications of MANETs are also envisioned in civilian usage [3]-[5], where nodes typically do not belong to a single authority and may not pursue a common goal. We refer to such networks as self-organized (self-organized) MANETs.

Before MANETs can be successfully deployed in a selforganized way, the issue of cooperation stimulation must be resolved first. In the literature, two types of schemes have been proposed to stimulate cooperation among selfish nodes: reputation-based schemes and payment-based schemes. In reputation schemes, such as [3], [4], [6], a node determines whether it should forward packets for other nodes or request other nodes to forward packets for it based on their past behaviors. In the payment-based schemes, such as [5], [7], a selfish node will forward packets for other nodes only if it can get some payment from those requesters as compensation.

Recently, some efforts have been made towards mathematically analyzing the cooperation in self-organized ad hoc networks using game theory, such as [8], [9]. In these existing game theoretic approaches, all of them have assumed perfect observation and most of them have not considered the effect of noise on the strategy design. However, in self-organized ad hoc networks, even when a node has decided to forward a packet for another node, this packet may still be dropped due to link breakage or transmission errors. Further, since central monitoring is in general not available in self-organized ad hoc networks, perfect public observation is either impossible or too expensive to be employed. Each node makes its decisions only based on its own past actions and imperfectly observed private information of its opponents.

In this paper we study the cooperation enforcement for self-organized mobile ad hoc networks with both noise and imperfect observation and focus on the most basic networking functioning, namely packet forwarding, in ad hoc networks. A belief-based packet forwarding approach is proposed to stimulate the packet-forwarding among nodes and maximize the expected payoff of each selfish node. Specifically, the repeated game model is applied to analyze the interactions among nodes. A formal belief system based on Bayes' rule is developed to assign and update beliefs of other nodes' continuation strategies for each node based on its private imperfect information. Further, we not only show that the packet forwarding strategy obtained from the proposed beliefbased framework achieves a sequential equilibrium that guarantees the strategy to be cheat-proof but also develop its performance bounds. The simulation results illustrate that the proposed belief-based packet forwarding approach can enforce the cooperation in the ad hoc networks with noise and imperfect observation with only a small performance degradation compared to the unconditionally cooperative outcomes.

The rest of this paper is organized as follows. The system model of self-organized ad hoc networks with noise and imperfect observation is presented in Section II. In Section III, we propose the belief-based packet forwarding framework and carry out the equilibrium and efficiency analysis. In Section IV, the belief-based multi-hop multi-node packet forwarding approach is developed. The simulation studies are provided in Section V. Finally, Section VI concludes this paper.

\section{SySTEM MODEL}

We consider self-organized ad hoc networks where nodes belong to different authorities and have different goals. Assume all nodes are selfish and rational, that is, their objective are to maximize their own payoff, not to cause damage to other nodes. Each node may act as a service provider: packets are scheduled to be generated and delivered to certain destinations; or act as a relay: forward packets for other nodes. The sender will get some payoffs if the packets are successfully delivered to the destination and the forwarding effort of relay nodes will also introduce certain costs. 


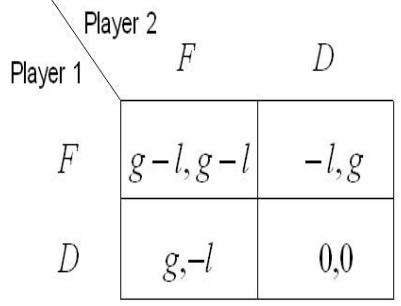

Fig. 1: Two-player packet forwarding game in strategic form.

In this paper we assume that some necessary traffic monitoring mechanisms, such as those described in [3], [5], [6], [10], will be launched by each node to keep tracking of its neighbors' actions. However, it is worth mentioning that we do not assume any public or perfect observation, where a public observation means that when an action happens, a group of nodes in the network will have the same observation, and perfect observation means all actions can be perfectly observed without any mistake. In ad hoc networks, due to its multi-hop nature and the lack of central monitoring mechanism, public observation is usually not possible. Meanwhile, to our best knowledge, these exist no such monitoring mechanisms in ad hoc networks which can achieve perfect observation. Instead, in this paper, we study the cooperation-enforcement strategies based on imperfect private observation. Here, private means that the observation of each node is only known to itself and won't or cannot be revealed to others. We focus on two scenarios causing imperfect observation in ad hoc networks. One scenario is that the outcome of the forwarding action may be packet dropping due to link breakage or transmission errors. The other scenario is that a node has dropped a packet but was observed as forwarding the packet, which may happen when the watchdog mechanism [3] is used and the node wants to cheat its previous node on the route.

\section{BELIEF-BASEd PACKET ForWARding FrameWORK}

Two-player packet forwarding game is studied in this section in attempt to shed light on the solutions to the more complicated multi-player case.

\section{A. Static and Repeated Packet-Forwarding Game Model}

We model the process of routing and packet-forwarding between a source node and a relay node as a game. The players of the game are the network nodes. There are two players in this game, denoted by $i \in I=\{1,2\}$. Each player is able to serve as the relay for the other player and needs the other player to forward packets for him based on current routing selection and topology. Each player chooses his action, i.e., strategy, $a_{i}$ from the action set $A=\{F, D\}$, where $F$ and $D$ are packet forwarding and dropping actions, respectively. Also, each player observes a private signal $\omega$ of the opponent's action from the set $\Omega=\{f, d\}$, where $f$ and $d$ are the cooperation and non-cooperation observations, respectively. Since the player's observation can not be perfect, the forwarding action $F$ of one player may be observed as $d$ by the other player due to link breakage or transmission error.
We let such probability be $p_{f}$. Also, the noncooperation action $D$ may be observed as the cooperation signal $f$ under certain circumstances. Without loss of generality, let the observation error probability be $p_{e}$ in our system, which is usually caused by cheating behaviors and the packet is actually dropped though forwarding signal $f$ is observed. For each node, the cost of forwarding a group of packets for the other node during one play is $\ell$, and the gain it can get for the packets that the other node has forwarded for it is $\tilde{g}$.

We first consider the packet forwarding as a static game, which is only played once. Given any action profile $a=$ $\left(a_{1}, a_{2}\right)$, we refer to $u(a)=\left(u_{1}(a), u_{2}(a)\right)$ as the expected payoff profile. Let $a_{-i}$ and $\operatorname{Prob}\left(\omega_{i} \mid a_{i}\right)$ be the action of the $i$ th player's opponent and the probability of having observation $\omega_{i}$ given $a_{-i}$, respectively. Then, $u_{i}(a)$ can be obtained as follows.

$$
u_{i}(a)=\sum_{\omega_{i} \in \Omega} \tilde{u}_{i}\left(a_{i}, \omega_{i}, a_{-i}\right) \cdot \operatorname{Prob}\left(\omega_{i} \mid a_{-i}\right),
$$

where $\tilde{u}_{i}$ is the $i$ th player's payoff depending on the action profile and his own observation. Then, calculating $u(a)$ for different strategy pairs, we have the strategic form of the static packet forwarding game as a matrix in Figure 1, where $g=$ $\left(1-p_{f}\right) \cdot \tilde{g}$.

To analyze the outcome of a static game, the Nash Equilibrium [11] is a well-known concept, which is the strategy, one for each other, such that no player has incentive to unilaterally change his action. The only Nash equilibrium of our two-player packet-forwarding game is the action profile $a^{*}=(D, D)$. But, the better cooperation payoff outcome $(g-\ell, g-\ell)$ of the cooperation action profile $\{F, F\}$ will not be practically realized in the static packet-forwarding game due to the greediness of the players. However, generally speaking, the above packet forwarding game will be played many times in real ad hoc networks. It is natural to extend the above static game model to a repeated game model. Basically, in the repeated games, the players face the same static game in every period, and the player's overall payoff is a weighted average of the payoffs in each stage over time.

Let $\omega_{i}^{t}$ be the privately observed signal of the $i$ th player in period $t$. Suppose that the game begins in period 0 with the null history $h^{0}$. In this game, a private history for player $i$ at period $t$, denoted by $h_{i}^{t}$, is a sequence of player $i$ 's past actions and signals, i.e., $h_{i}^{t}=\left\{a_{i}^{\tau}, \omega_{i}^{\tau}\right\}_{\tau=1}^{t-1}$. Denote the infinite packetforwarding repeated game with imperfect private histories by $G(p, \delta)$, where $p=\left(p_{f}, p_{e}\right), \delta \in(0,1)$ is the discount factor. Assume that $p_{f}<1 / 2$ and $p_{e}<1 / 2$. Then, the overall discounted payoff for player $i \in I$ is defined as follows [11].

$$
U_{i}(\delta)=(1-\delta) \sum_{t=0}^{\infty} \delta^{t} u_{i}^{t}\left(a_{1}^{t}\left(h_{1}^{t}\right), a_{2}^{t}\left(h_{2}^{t}\right)\right) .
$$

Folk Theorems for infinite repeated games [11] assert that there exists a $\hat{\delta}<1$ such that any feasible and individually rational payoff can be enforced by an equilibrium for all $\delta \in(\hat{\delta}, 1)$ based on the public information shared by players. However, one crucial assumption for the Folk Theorems is that 
players share common information about each other's actions. In contrast, the nature of our repeated packet forwarding game for self-organized ad hoc networks determines that the nodes' behaviorial strategies can only rely on the private information histories including their own past actions and imperfectly observed signals. A seemingly minor game-setting change from the public observation to the private observation due to noise and imperfect observation will make a substantial difference in analyzing the efficiency of the packet-forwarding game. In the situations of imperfect private observation, each node must conduct statistical inference to detect potential deviations and estimate what others are going to do next, which can become extremely complex due to the imperfect observation [12], [13].

\section{B. Belief-Based Packet Forwarding Approach}

In order to have an efficient and robust forwarding strategy based on each node's own observation and actions, we propose a belief-based packet forwarding approach enlightened by [13].

First, we define two strategies, i.e., $\sigma_{F}$ and $\sigma_{D}$. Let $\sigma_{F}$ be the trigger cooperation strategy, which means that the player forwards packets at current stage, and at the next stage the player will continue to forward packets only if it observes the other player's forwarding signal $f$. Let $\sigma_{D}$ be the defection strategy, which means that the player always drops packets regardless of its observation history. Such strategies are also called continuation strategies [13]. Since both of the two strategies also determine the player's following actions at every private history, the strategy path and expected future payoffs caused by any pair of the two strategies are fully specified. Let $V_{\alpha, \beta}(p, \delta), \alpha, \beta \in\{F, D\}$ denote the repeated game payoff of $\sigma_{\alpha}$ against $\sigma_{\beta}$, which can be illustrated by the following Bellman equations [14] for different pairs of continuation strategies.

$$
\begin{aligned}
& V_{F F}=(1-\delta)(g-\ell)+\delta\left(\left(1-p_{f}\right)^{2} V_{F F}+\right. \\
&\left.p_{f}\left(1-p_{f}\right) V_{F D}+p_{f}\left(1-p_{f}\right) V_{D F}+p_{f}^{2} \cdot V_{D D}\right), \\
& V_{F D}=-(1-\delta) \ell+\delta\left(\left(1-p_{f}\right)\left(1-p_{e}\right) V_{D D}+\right. \\
&\left.p_{f}\left(1-p_{e}\right) V_{D D}+p_{e}\left(1-p_{f}\right) V_{F D}+p_{f} p_{e} V_{F D}\right), \\
& V_{D F}=(1-\delta) g+\delta\left(\left(1-p_{f}\right)\left(1-p_{e}\right) V_{D D}+\right. \\
&\left.p_{e}\left(1-p_{f}\right) V_{D F}+p_{f}\left(1-p_{e}\right) V_{D D}+p_{e} p_{f} V_{D F}\right), \\
& V_{D D}=(1-\delta) \cdot 0+\delta\left(\left(1-p_{e}\right)^{2} V_{D D}+\right. \\
&\left.p_{e}\left(1-p_{e}\right) V_{D D}+p_{e}\left(1-p_{e}\right) V_{D D}+p_{e}^{2} \cdot V_{D D}\right) .
\end{aligned}
$$

Note that the first term in the above equations represents the normalized payoffs of current period, while the second term illustrates the expected continuation payoffs considering four possible outcomes due to the imperfect observation. By solving the above equations, $V_{\alpha, \beta}(p, \delta)$ can be easily obtained. Then, we have $V_{D D}>V_{F D}$, for any $\delta, p$. Furthermore, if $\delta>\delta_{0}$, then $V_{F F}>V_{D F}$, where $\delta_{0}$ can be calculated as

$$
\delta_{0}=\frac{\ell}{\left(1-p_{f}-p_{e}\right) g-\left[p_{f}\left(1-p_{f}\right)-p_{e}\right] \ell},
$$

TABLE I: Belief-based Two-player Packet Forwarding Algorithm

\begin{tabular}{|l|}
\hline 1. Initialize using system parameter configuration $\left(\delta, p_{e}, p_{f}\right)$ : \\
Node $i$ initializes his belief $\mu_{i}^{1}$ of the other node as $\pi(\delta, p)$ \\
and chooses the forwarding action in period 1 with probability $\pi(\delta, p)$. \\
\hline 2. Belief update based on the private history: \\
Update each node's belief $\mu_{i}^{t-1}$ into $\mu_{i}^{t}$ using (10-13) according to \\
different realizations of private history. \\
\hline 3. Optimal Decision of the player's next move: \\
If the continuation belief $\mu_{i}^{t}>\pi$, node $i$ plays $\sigma_{F} ;$ \\
If the continuation belief $\mu_{i}^{t}<\pi$, node $i$ plays $\sigma_{D} ;$ \\
If the continuation belief $\mu_{i}^{t}=\pi$, node $i$ plays either $\sigma_{F}$ or $\sigma_{D}$. \\
\hline 4. Iteration: \\
Let $t=t+1$, then go back to Step 2.
\end{tabular}

Note that this constraint of $\delta$ will prevent trivial defection outcomes. Suppose that player $i$ believes that his opponent is playing either $\sigma_{F}$ or $\sigma_{D}$, and is playing $\sigma_{F}$ with probability $\mu$. Then the difference between his payoff of playing $\sigma_{F}$ and the payoff of playing $\sigma_{D}$ is given by

$$
\triangle V(\mu ; \delta, p)=\mu \cdot\left(V_{F F}-V_{D F}\right)-(1-\mu) \cdot\left(V_{D D}-V_{F D}\right) .
$$

Hence $\triangle V(\mu)$ is increasing and linear in $\mu$ and there is a unique value $\pi(p, \delta)$ to make it zero, which can be obtained as follows.

$$
\pi(\delta, p)=\frac{-V_{F D}(\delta, p)}{V_{F F}(\delta, p)-V_{D F}(\delta, p)-V_{F D}(\delta, p)},
$$

where $\pi(p, \delta)$ is defined so that player $i$ has no preference in choosing $\sigma_{F}$ or $\sigma_{D}$ when player $j$ plays $\sigma_{F}$ with probability $\pi(\delta, p)$ and $\sigma_{D}$ with probability $1-\pi(\delta, p)$. For simplicity, $\pi(\delta, p)$ may be denoted as $\pi$ in the following parts under the circumstances with no confusion. Intuitively, if node $i$ holds the belief that the other node will help him to forward the packets with a probability smaller than $1 / 2$, node $i$ is inclined not to forward packets for the other node. Considering such situation, we let $\delta$ be such that $\pi(\delta, p)>1 / 2$.

It is worth mentioning that equation (8) is applicable to any period. Thus, if a node's belief of an opponent's continuation strategy being $\sigma_{F}$ is $\mu$, in order to maximize its expected continuation payoff, the node prefers $\sigma_{F}$ to $\sigma_{D}$ if $\mu>\pi$ and prefers $\sigma_{D}$ to $\sigma_{F}$ if $\mu<\pi$. Given any initial belief $\mu$, the $i$ th player's new belief when he takes action $a_{i}$ and receives signal $\omega_{i}$ can be defined using the Bayes' rule [11] as follows.

$$
\begin{gathered}
\mu\left(h_{i}^{t-1},(F, f)\right)=\frac{\mu\left(h_{i}^{t-1}\right)\left(1-p_{f}\right)^{2}}{\mu\left(h_{i}^{t-1}\right)\left(1-p_{f}\right)+p_{e} \cdot\left(1-\mu\left(h_{i}^{t-1}\right)\right)}, \\
\mu\left(h_{i}^{t-1},(F, d)\right)=\frac{\mu\left(h_{i}^{t-1}\right)\left(1-p_{f}\right) \cdot p_{f}}{\mu\left(h_{i}^{t-1}\right) \cdot p_{f}+\left(1-p_{e}\right) \cdot\left(1-\mu\left(h_{i}^{t-1}\right)\right)}, \\
\mu\left(h_{i}^{t-1},(D, f)\right)=\frac{\mu\left(h_{i}^{t-1}\right)\left(1-p_{f}\right) \cdot p_{e}}{\mu\left(h_{i}^{t-1}\right) \cdot\left(1-p_{f}\right)+p_{e} \cdot\left(1-\mu\left(h_{i}^{t-1}\right)\right)}, \\
\mu\left(h_{i}^{t-1},(D, d)\right)=\frac{\mu\left(h_{i}^{t-1}\right) p_{f} \cdot p_{e}}{\mu\left(h_{i}^{t-1}\right) \cdot p_{f}+\left(1-p_{e}\right) \cdot\left(1-\mu\left(h_{i}^{t-1}\right)\right)} .
\end{gathered}
$$

Based on the above discussion, we propose a two-player belief-based packet forwarding algorithm shown in Table I. 


\section{Efficiency Analysis of the Belief-Based Packet Forwarding Framework}

In this part, we show that the behaviorial strategy obtained from the proposed algorithm with well-defined belief systems is a sequential equilibrium and further analyze its performance bounds.

First, we briefly introduce the equilibrium concepts of the repeated games with imperfect information. As for the infinitely repeated game with perfect information, the Nash Equilibrium concept is a useful concept for analyzing the game outcomes. But, since the threats in Nash equilibria may not be credible and become empty threats, the subgame perfect equilibrium [11] is defined to eliminate those equilibria in which the players' threats are incredible. However, the above equilibrium criteria for the infinitely repeated game require that perfect information can be obtained for each player. In our packet forwarding game, each node is only able to have his own strategy history and form the beliefs of other nodes' future actions through imperfect observation.

Sequential Equilibrium [11] is a well-defined counterpart of subgame perfect equilibrium under such circumstance, which guarantees that any deviations will be unprofitable.

In our packet-forwarding game with private history and observation, the proposed strategy with belief-system can be denoted as $\left(\sigma^{*}, \mu\right)$, where $\mu=\left\{\mu_{i}\right\}_{i \in I}$ and $\sigma^{*}=\left\{\sigma_{i}^{*}\right\}_{i \in I}$. By studying (10), we find that there exists a point $\phi$ such that $\mu\left(h_{i}^{t-1},(F, f)\right)<\mu\left(h_{i}^{t-1}\right)$ as $\mu\left(h_{i}^{t-1}\right)>\phi$ while $\mu\left(h_{i}^{t-1},(F, f)\right)>\mu\left(h_{i}^{t-1}\right)$ as $\mu\left(h_{i}^{t-1}\right)<\phi$. Here, $\phi$ can be calculated as $\phi=\left[\left(1-p_{f}\right)^{2}-p_{e}\right] /\left(1-p_{f}-p_{e}\right)$. It is easy to show that $\mu\left(h_{i}^{t-1},\left(a_{i}, \omega_{i}\right)\right)<\mu\left(h_{i}^{t-1}\right)$ when $(F, d)$, $(D, f)$ and $(D, d)$ are reached. Since we initialize the belief with $\pi$, we have $\mu_{i}^{t} \leq \phi$ after any belief-updating operation if $\pi<\phi$. Considering the belief updating in the scenario that $\pi \geq \phi$ becomes trivial, we assume $\pi<\phi$, thus $\mu_{i}^{t} \in[0, \phi]$ and $\phi>1 / 2$. Then, let the proposed packet-forwarding strategy profile $\sigma^{*}$ be defined as: $\sigma_{i}^{*}\left(\mu_{i}\right)=\sigma_{F}$ if $\mu_{i}>\pi$ and $\sigma_{i}^{*}\left(\mu_{i}\right)=\sigma_{D}$ if $\mu_{i}<\pi$; If $\mu_{i}=\pi$, the node forwards packets with probability $\pi$ and drops them with probability $1-\pi$. Similar to [13], we have the following two theorems.

Theorem 1: The proposed strategy profile $\sigma^{*}$ with the belief system $\mu$ from Table $\mathrm{I}$ is a sequential equilibrium for $\pi \in$ $(1 / 2, \phi)$.

Theorem 2: Given $g$ and $\ell$, there exist $\tilde{\delta} \in(0,1)$ and $\tilde{p}$ for any small positive $\tau$ such that the average payoff of the proposed strategy $\sigma^{*}$ in the packet-forwarding repeated game $G(p, \delta)$ is greater than $g-\ell-\tau$ if $\delta>\tilde{\delta}$ and $p_{e}, p_{f}<\tilde{p}$.

Theorem 1 shows that the strategy profile $\sigma^{*}$ and the belief system $\mu$ obtained from the proposed algorithm is a sequential equilibrium, which not only responds optimally at every history but also has consistency on zero-probability histories. Thus, the cooperation can be enforced using our proposed algorithm since the deviation will not increase the players' payoffs. Then, Theorem 2 addresses the efficiency of the equilibrium and shows that when the $p_{e}$ and $p_{f}$ are small enough, our proposed strategy approaches the coop- erative payoff $g-\ell$. However, in real ad hoc networks, a more useful and important measurement is the performance bounds of the proposed strategy given some fixed $p_{e}$ and $p_{f}$ values. We further develop the following theorem studying the lower bound and upper bound of our strategy to provide a performance guideline. In order to model the prevalent data application in current ad hoc networks, we assume that the game discount factor is very close to 1 .

Theorem 3: Given the fixed $\left(p_{e}, p_{f}\right)$ and discount factor of the repeated game $\delta_{G}$ close to 1 , the payoff of the proposed algorithm in Table I is upper bounded by

$$
\bar{U}=(1-\kappa) \cdot(g-\ell),
$$

where

$$
\kappa=\frac{p_{f} \cdot\left[g\left(1-p_{f}\right)+\ell\right]}{\left(1-p_{f}-p_{e}\right)(g-\ell)} .
$$

The lower bound of the performance will approach the upper bound when the discount factor of the repeated game $\delta_{G}$ approaches 1 if the packet forwarding game is divided into $N$ sub-games as follows: the first sub-game is played in period $1, N+1,2 N+1, \ldots$ and the second sub-game is played in period $2, N+2,2 N+2, \ldots$, and so on. The optimal $N$ is

$$
N=\left\lfloor\log \underline{\delta} / \log \delta_{G}\right\rfloor,
$$

where $\underline{\delta}=\ell /\left\{\left[\left(1-p_{f}\right)^{2}-p_{e}\right] \cdot g+\ell \cdot p_{e}\right\}$. The proposed strategy is played in each sub-game with equivalent discount factor $\delta_{G}^{N}$.

In the above theorem, by introducing the idea of dividing the original game into several sub-games, even if the outcomes of some sub-games become the non-cooperation case due to the observation errors, cooperation plays can still continue in other sub-games to increase the total payoff. Note that due to the limited space, the proofs of theorems are omitted here, which can be found in [15].

\section{Belief-Based Multi-Node Multi-HoP PACKET FORWARDING}

\section{A. Multi-Node Multi-Hop Game Model}

In this section, we consider self-organized ad hoc networks where nodes can move freely inside a certain area. For each node, packets are scheduled to be generated and sent to certain destinations. Different from the two-player packet forwarding game, the multi-player packet forwarding game studies multihop packet forwarding which involves the interactions and beliefs of all the nodes on the route. Before studying the beliefbased strategy in this scenario, we first model the multi-player packet forwarding game as follows:

- There are $M$ players in the game, which represent $M$ nodes in the network. Denote the player set as $I_{M}=$ $\{1,2, \ldots, M\}$.

- For each player $i \in I_{M}$, he has groups of packets to be delivered to certain destinations at different time.

- For each player $i \in I_{M}$, forwarding a packet for another player will incur some cost $\ell$. 


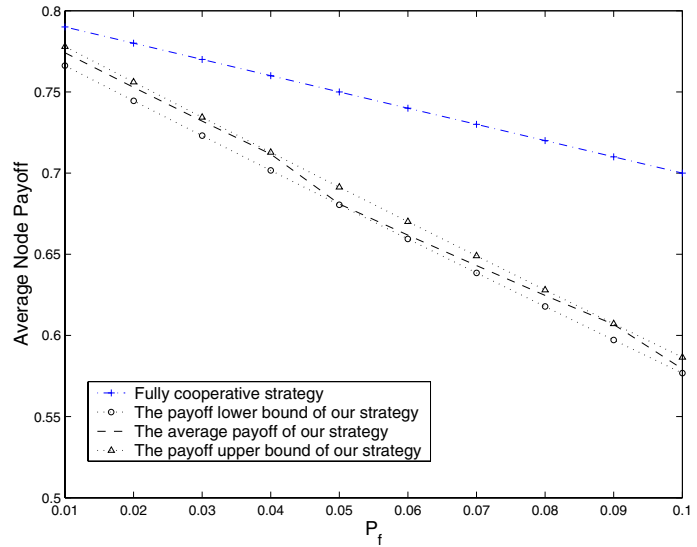

Fig. 2: The average payoffs of the cooperative strategy and proposed strategy.

- Due to the multi-hop nature of ad hoc networks, the destination player $j$ may not lie in the sending player $i$ 's direct transmission range. Player $i$ needs to not only find the possible routes leading to the destination (i.e., route discovery), but also choose an optimal route from the routing candidates (i.e., route selection).

- Each player only knows his own past action and imperfect observation of other players' action. Note that the information history consisting of the above two parts is private to each player.

Similar to [8], we assume the network operates in discrete time. In each time slot, one node is randomly selected from the $M$ nodes as the sender. The probability that the sender finds $r$ possible routes is given by $q_{r}(r)$ and the probability that each route needs $\hbar$ hops is given by $q_{\hbar}(\hbar)$ (assume at lease one hop is required in each time slot). Note that the $\hbar$ relays on each route are selected from the rest of nodes with equal probability and $\hbar \leq\lfloor g / \ell\rfloor$. Assume each routing session lasts for one slot and the routes remain unchanged within each time slot. In our study, we consider that delicate traffic monitoring mechanisms such as receipt-submission approaches [5] are in place, hence the sender is able to have the observation of each node on the forwarding route.

\section{B. Belief-Based Strategy for Multi-hop Packet Forwarding}

In this part, we develop efficient belief-based strategies for multi-hop packet forwarding games based on the proposed two-player approach. Let $\omega_{i j}, \mu_{i j}$ and $h_{i j}$ denote the sending player $i$ 's observation, belief value and the private history on the forwarding player $j$, respectively. The proposed forwarding strategy for the multi-player case is illustrated as follows.

Belief-Based Multi-hop Packet Forwarding (BMPF) Strategy: In the multi-node multi-hop packet forwarding game, given the discount factor $\delta_{G}$ and $p=\left(p_{e}, p_{f}\right)$, the sender and relay nodes act as follows during different phases of routing process.

- Game partition and belief initialization: Partition the original game into $N$ sub-games according to (16). Then, each node initializes its belief of other nodes as $\pi\left(\delta_{G}^{N}, p\right)$ and forwards packets with probability $\pi\left(\delta_{G}^{N}, p\right)$

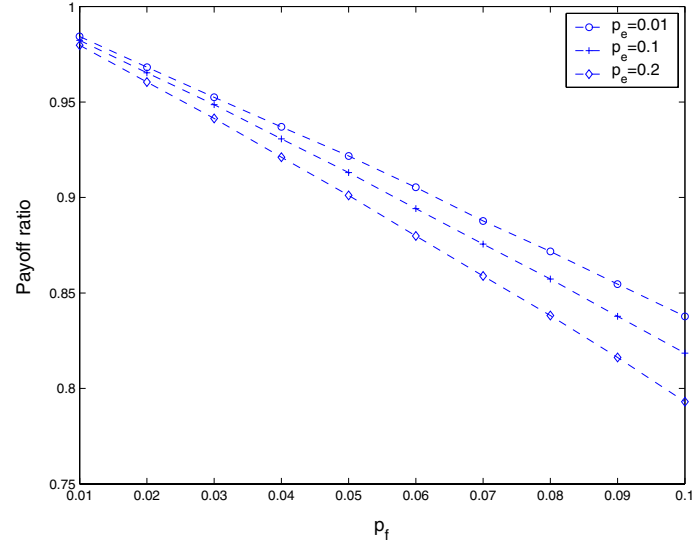

Fig. 3: Payoff ratios of the proposed strategy to the cooperative strategy.

- Route participation: The selected relay node on each route participates in the routing if and only if its belief of the sender is greater than $\pi$.

- Route selection: The sender selects the route with the largest $\mu_{i}=\prod_{j \in R_{i}} \mu_{i j}$ from the route candidates.

- Packet forwarding: The sender updates its belief of each relay node's continuation strategy using (10)-(13) and decide the following actions based on its belief.

Based on Theorem 1, it is straight-forward to show that BMPF Strategy designed for multi-node multi-hop packet forwarding games is also a sequential equilibrium. Here, in order to calculate the equivalent two-player expected gain $g$ in Table I, we need to consider the routing statistics such as $q_{r}(r)$ and $q_{\hbar}(\hbar)$ to deal with the error propagation and routing diversity.

\section{Simulation Studies}

In this section, we investigate the cooperation enforcement results of our proposed belief-based packet forwarding approach by simulation.

We first focus our simulation studies on one-hop packet forwarding scenarios in ad hoc networks, where the twoplayer belief-based packet forwarding approach can be directly applied to. Let $M=100, g=1$ and $\ell=0.2$ in our simulation. For comparison, we define the cooperative strategy, which assumes every node will unconditionally forwarding packets with no regard to other nodes' past behaviors. Such cooperative strategy is not implementable in self-organized ad hoc networks. But it can serve as a loose upper bound of the performance of the proposed strategy and determine the performance loss of cooperation enforcement due to noise and imperfect observation.

Figure 2 shows the average payoff and performance bounds of the proposed belief-based strategy for different $p_{f}$ by comparing them with the cooperative payoff. Note that $p_{e}=$ 0.01 and $\delta_{G}=0.99$. It can be seen from Figure 2 that our proposed approach can enforce cooperation with only small performance loss compared to the unconditionally cooperative payoff. Further, this figure shows that the average payoff of our proposed strategy satisfies the theoretical payoff bounds 


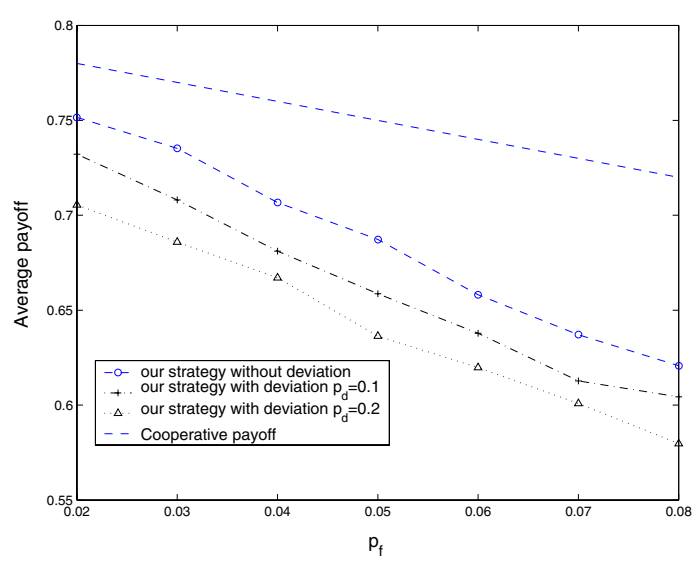

Fig. 4: Payoff comparison of the proposed strategy and deviating strategies.

developed in Theorem 3. The fluctuation of the payoff curve of our strategy is because that only integer number of sub-games can be partitioned from the original game. Figure 3 shows the ratio of the payoffs of our strategy to those of the cooperative strategy for different $p_{e}$ and $p_{f}$. Here we let $\delta_{G}=0.999$ to approach the payoff upper bound. It can be seen from Figure 3 that even if $p_{f}$ is as large as 0.1 due to link breakage or transmission error, our cooperation enforcement strategy can still achieve as high as $80 \%$ of the cooperative payoff.

In order to show that the proposed strategy is cheat-proof among selfish users, we define the deviation strategies for comparison. The deviation strategies differ from the proposed strategy only when the continuation strategy $\sigma_{F}$ and observation $F$ are reached. The deviation strategies will play $\sigma_{D}$ with deviating probability $p_{d}$ instead of playing $\sigma_{F}$ as the proposed strategy specifies. Figure 4 compares the nodes' average payoffs of the proposed strategy, cooperative strategy and deviation strategies with different deviating probabilities. Note that $\delta_{G}=0.999$ and $p_{e}=0.1$. This figure shows that the proposed strategy has much better payoffs than the deviating strategies.

Then, we study the performance of the proposed multi-hop multi-node packet forwarding approach. Let $q_{r}(1)=q_{r}(2)=$ $1 / 2, q_{\hbar}(2)=q_{\hbar}(3)=1 / 2$ and $\delta_{G}=0.999$. We compare the payoff of our approach with that of the cooperative one in Figure 5. Note that multi-hop forwarding will incur more costs to the nodes since one successful packet delivery involves the packet forwarding efforts of many relay nodes. Also, the noise and imperfect observation will have more impact on the performance as each node's incorrect observation will affect the payoffs of all other nodes on the selected route. We can see from Figure 5 that our proposed strategy still maintains high payoffs even when the environment is noisy and the observation error is large.

\section{CONCLUSION}

In this paper, we study the cooperation enforcement in self-organized ad hoc networks with noise and imperfect observation. By modeling the basic network function, packet forwarding, as a repeated game with imperfect information,

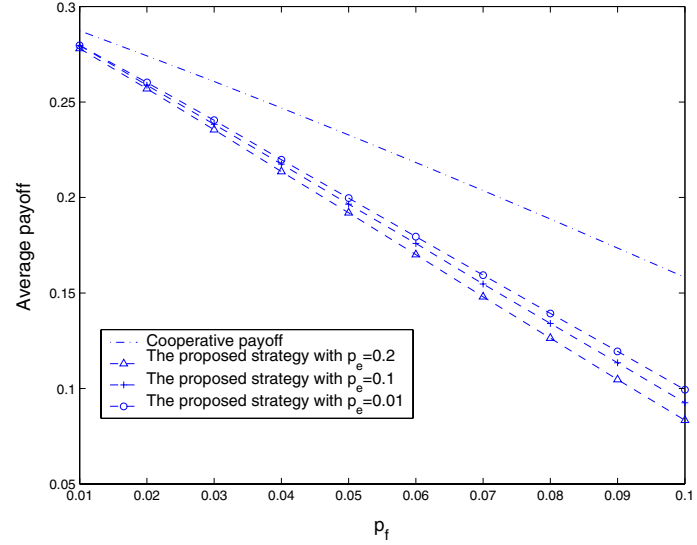

Fig. 5: Average payoffs of the proposed strategy and deviating strategies in multi-node multi-hop scenarios.

we develop the belief-based packet forwarding framework to enforce cooperation in the scenarios of noise and imperfect observation. We show that the behaviorial strategy with welldefined belief system from the proposed approach can not only achieve the sequential equilibrium but also maintain high payoffs for both two-player and multi-player cases. The simulation results illustrate that the proposed belief-based packet forwarding approach achieves stable and near-optimal equilibrium in the ad hoc networks with imperfect observation.

\section{REFERENCES}

[1] C. Perkins, Ad Hoc Networking, Addison-Wesley, 2000.

[2] C. K. Toh, Ad Hoc Mobile Wireless Networks: Protocols and Systems, Prentice Hall PTR, 2001.

[3] S. Marti, T. J. Giuli, K. Lai, and M. Baker, "Mitigating Routing Misbehavior in Mobile Ad Hoc Networks," in ACM Mobicom 2000, August 2000, pp. 255-265.

[4] P. Michiardi and R. Molva, "CORE: a COllaborative REputation Mechanism to Enforce Node Cooperation in Mobile Ad Hoc Networks," in IFIP - Communications and Multimedia Security Conference, 2002.

[5] S. Zhong, J. Chen, and Y. R. Yang, "SPRITE: A Simple, CheatProof, Credit-Based System for Mobile Ad-Hoc Networks," in IEEE INFOCOM, 2003.

[6] W. Yu and K. J. R. Liu, "Attack-Resistant Cooperation Stimulation in Autonomous Ad Hoc Networks," to appear in IEEE Journal on Selected Areas in Communications, special issue in Autonomic Communication Systems, Dec. 2005.

[7] L. Buttyan and J. P. Hubaux, "Enforcing Service availability in mobile Ad-hoc Network," in ACM MobiHOC'O0, August 2000.

[8] V. Srinivasan, P. Nuggehalli, C. F. Chiasserini, and R. R. Rao, "Cooperation in Wireless Ad Hoc Networks," in IEEE INFOCOM, 2003.

[9] M. Felegyhazi, J.-P. Hubaux, and L. Buttyan, "Nash Equilibria of Packet Forwarding Strategies in Wireless Ad Hoc Networks," to appear in IEEE Transactions on Mobile Computing, 2005.

[10] W. Yu, Y. Sun, and K. J. R. Liu, "HADOF: Defense against Routing Disruptions in Mobile Ad Hoc Networks," in Proc. of IEEE INFOCOM'05, 2005.

[11] M. J. Osborne and A. Rubinstein, A Course in Game Theory, The MIT Press, Cambridge, Massachusetts, 1994.

[12] T. Sekiguchi, "Efficiency in repeated prisoner's dilemma with private monitoring," Journal of Economic Theory, vol. 76, pp. 345-361, 1997.

[13] V. Bhaskar and I. Obara, "Belief-based equilibria in the repeated prisoners' dilemma with private monitoring," Journal of Economic Theory, vol. 102, pp. 40-69, 2002.

[14] D. Bertsekas, Dynamic Programming and Optimal Control, vol. 1,2, Athena Scientific, Belmont, MA, Second edition, 2001.

[15] Z. Ji, W. Yu, and K. J. R. Liu, "Cooperation enforcement in autonomous mobile ad hoc networks under noisy and imperfect observations," submitted to IEEE Transactions on Mobile Computing, 2005. 